\title{
High prevalence of risk factors for cardiovascular disease in parents of IDDM patients with albuminuria
}

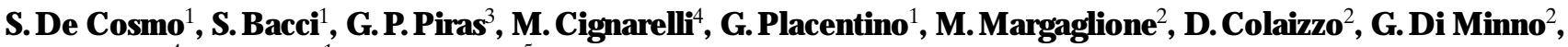 \\ R .G iorgino ${ }^{4}$, A . L iuzzi ${ }^{1}$, G .C.V iberti $^{5}$ \\ ${ }^{1}$ Division of Endocrinology, IRCCS “Casa Sollievo della Sofferenza” San Giovanni Rotondo, Italy \\ ${ }^{2}$ Unit for Atherosclerosis and Thrombosis IRCCS "Casa Sollievo della Sofferenza”, San Giovanni Rotondo, Italy \\ ${ }^{3}$ Diabetes Unit, Ospedale "San Michele", Cagliari, Italy \\ ${ }^{4}$ Istituto di Clinica Medica, Policlinico, Bari, Italy \\ ${ }^{5}$ Unit for Metabolic Medicine, UMDS Guy's Hospital, London, UK
}

Summary Life expectancy is shorter in the subset of insulin-dependent diabetic (IDDM) patients who are susceptible to kidney disease. Familial factors may be important. In this study the prevalence of cardiovascular disease mortality and morbidity and of risk factors for cardiovascular disease was compared in the parents of 31 IDDM patients with elevated albumin excretion rate (AER $>45 \mu \mathrm{g} / \mathrm{min}$; group A) with that of parents of 31 insulin-dependent diabetic patients with normoalbuminuria (AER $<20 \mu \mathrm{g} / \mathrm{min}$; group B). The two diabetic patient groups were matched for age and duration of disease. Information on deceased parents was obtained from death certificates and clinical records and morbidity for cardiovascular disease was ascertained using the World Health Organization questionnaire and Minnesota coded ECG. Hyperlipidaemia was defined as serum cholesterol higher than $6 \mathrm{mmol} / \mathrm{l}$ and/or plasma triglycerides higher than $2.3 \mathrm{mmol} / \mathrm{l}$ and/or lipid lowering therapy; arterial hypertension as systolic blood pressure higher than $140 \mathrm{mmHg}$ and/or diastolic blood pressure higher than $90 \mathrm{mmHg}$ and/or antihypertensive treatment. The percentage of dead parents was similar in the two groups (26 vs $20 \%$ for parents of group A vs group B, respectively), but the parents of the diabetic patients with elevated AER had died at a younger age $(58 \pm 10$ vs $70 \pm 14$ years; $p<0.05)$. Parents of diabetic patients with nephropathy had a more than three times greater frequency of combined mortality and morbidity for cardiovascular disease than that of the parents of diabetic patients without nephropathy ( 26 vs $8 \%$; odds ratio $3.96,95 \%$ CI 1.3 to $12.2 ; p<0.02)$. Living parents of group A had a higher prevalence of arterial hypertension (42 vs $14 \% \mathrm{p}<0.01$ ) and hyperlipidaemia (49 vs $26 \% p<$ 0.05 ) as well as higher levels of lipoprotein (a) [median (range) 27.2 (1-107) vs $15.6(0.2-98) \mathrm{mg} / \mathrm{dl}$; $p<0.05]$. They also had reduced insulin sensitivity [insulin tolerance test: median (range) $\mathrm{K}_{\text {itt }}$ index: 3.7 (0.7-6.2) vs $4.8(0.7-6.7) \%$ per min; $p<0.05]$. In the families of IDDM patients with elevated AER there was a higher frequency of risk factors for cardiovascular disease as well as a predisposition to cardiovascular disease events. This may help explain, in part, the high prevalence of cardiovascular disease mortality and morbidity in those IDDM patients who develop nephropathy. [Diabetologia (1997) 40: 1191-1196]

Keywords Insulin-dependent diabetes mellitus, proteinuria, insulin resistance, hyperlipidaemia, cardiovascular disease.
Received: 4 March 1997 and in revised form: 9 May 1997

Corresponding author: Dr. S. De Cosmo, Divisione di Endocrinologia, IRCCS "Casa Sollievo della Sofferenza, " I-71 013 San Giovanni Rotondo (FG), Italy

A bbreviations: IDDM, Insulin-dependent diabetes mellitus; NIDDM, non-insulin-dependent diabetes mellitus; AER, albumin excretion rate; ITT, insulin tolerance test.
In insulin-dependent diabetic (IDDM) patients survival is significantly affected by the development of nephropathy which leads to renal failure and increased cardiovascular mortality [1].

Raised albumin excretion rate (AER) per se is strongly related to early cardiovascular morbidity and mortality both in insulin-dependent and non-insulin-dependent (NIDDM) diabetic patients [2, 3]. Moreover IDDM patients with microalbuminuria, a 
group at high risk of renal failure, are insulin resistant [4] and have lipid abnormalities [5], both risk factors for cardiovascular disease. The mechanisms of interaction between metabolic abnormalities and clinical manifestations of disease are not fully elucidated but there is evidence that the clinical outcome of cardiovascular disease is not simply the result of glycaemic control, renal failure or renal replacement therapy. Other factors appear to be of importance. Diabetic nephropathy clusters in families [6] and furthermore non-diabetic relatives of IDDM patients with nephropathy have a higher prevalence of arterial hypertension [7] and cardiovascular disease [8]. That familial factors contribute to the risk of nephropathy in diabetes is supported by the observation that certain intermediary phenotypes for hypertension and cardiovascular disease, such as sodium-lithium countertransport [9], whose activity is predominantly genetically determined, operate at elevated rates in IDDM patients with albuminuria. Whether the families of IDDM patients with nephropathy share metabolic and biochemical risk factors for cardiovascular disease remains largely unexplored. In a preliminary study we have observed higher fasting insulin concentrations, serum cholesterol and apolipoprotein B levels and low-density-lipoprotein/high-density-lipoprotein cholesterol ratio in non-diabetic first degree relatives of IDDM patients with microalbuminuria [10].

Therefore the aim of this study was to investigate whether metabolic and biochemical risk factors for cardiovascular disease in parents were related to the development of nephropathy in diabetic offspring and might explain the excess cardiovascular disease risk.

\section{Subjects and methods}

Patients with nephropathy. Patients with IDDM as indicated by disease onset before age 30 years, evidence of ketosis at diagnosis and absolute need of continued insulin therapy within 6 months of diagnosis, who were less than 60 years old and were attending the diabetic clinics of Ospedale "Casa Sollievo della Sofferenza", San Giovanni Rotondo; Clinica Medica, University of Bari; and Ospedale San Michele, Cagliari between July 1993 and September 1994 were screened for the study. Patients were considered to have diabetic nephropathy if their urinary AER was greater than $45 \mu \mathrm{g} / \mathrm{min}$ in at least three sterile overnight urine specimens collected over a 3 month period, they had had diabetes for at least 10 years, they had diabetic retinopathy, and they did not have heart failure or another renal disease. The level of AER of $45 \mu \mathrm{g} / \mathrm{min}$ was chosen to ensure significant renal histological lesions $[11,12]$ and to exclude lower levels of microalbuminuria which may not progress.

All 31 patients who fulfilled the entry criteria were included in this group (group A). Antihypertensive therapy was used in $71 \%$ of patients.

Patients without nephropathy. Of the patients with IDDM and without raised AER (AER $<20 \mu \mathrm{g} / \mathrm{min}$ ), who were less than 60 years old, had a diabetes duration greater than 15 years
Table 1. Clinical features of IDDM patients with or without nephropathy

\begin{tabular}{lll}
\hline Characteristic & Nephropathy & No nephropathy \\
\hline Sex (male/female) & $16 / 15$ & $16 / 15$ \\
Age (years) $^{\mathrm{a}}$ & $35(25-56)$ & $35(25-52)$ \\
Duration of diabetes (years) $^{\mathrm{a}}$ & $23(10-38)$ & $20.3(16-49)$ \\
${\text { Albumin excretion rate }(\mu \mathrm{g} / \mathrm{min})^{\mathrm{b}}}^{\mathrm{a}}$ & $460(46-2500)$ & $12(2-18)$ \\
Systolic blood pressure $(\mathrm{mm} \mathrm{Hg})$ & $140 \pm 16$ & $136 \pm 16$ \\
Diastolic blood pressure $(\mathrm{mm} \mathrm{Hg})^{8}$ & $84 \pm 8$ & $80 \pm 8$ \\
Hypertension $(\%)$ & 71 & $10^{\mathrm{c}}$
\end{tabular}

Data are mean \pm SD

${ }^{\mathrm{a}}$ Mean (range); ${ }^{\mathrm{b}}$ geometric mean (range);

${ }^{c} p<0.001$

and were attending the clinics between July 1993 and September 1994, 68 met the inclusion criteria for the study. From these eligible patients 31 were selected as a control group (group B) to be as closely comparable as possible to the patients with nephropathy with respect to age, duration of diabetes and sex. Three patients were on antihypertensive therapy. Table 1 shows the clinical features of the two groups.

Parents of the patients. Information concerning a parental history of cardiovascular disease was sought for all diabetic patients. We obtained information for all parents of the patients with nephropathy and for all parents of the patients without nephropathy. Information on deceased parents was taken from death certificates obtained from the Registry Office of the Municipality of residence. Causes of death were certified by the international code:ICD-9 [13]. Where possible the information from death certificates was supplemented by data from clinical records. A record of myocardial infarction, heart failure, ruptured aortic aneurysm, or cerebrovascular accident was taken as evidence that cardiovascular disease was a direct cause of death. All the parents who were alive at the time of the study were invited to a metabolic ward where they were interviewed in a standardized manner using the World Health Organization (WHO) questionnaire for cardiovascular disease to which a question concerning stroke was added $[14,15]$. Ascertainment in this study was therefore $100 \%$. Previous hospital-verified myocardial infarction, previous coronary bypass surgery or angioplasty and physician-diagnosed angina pectoris or possible infarction by WHO questionnaire were taken as evidence of coronary heart disease. Hospital-verified stroke or transient ischaemic attacks verified by neurologist were taken as evidence of presence of cerebrovascular disease. Further questions were asked concerning diabetes, arterial hypertension, dyslipidaemia, drugs taken, smoking habits and physical activity. A diagnosis of diabetes was made if the subjects were receiving specific treatment for this condition. Parents were considered smokers if they were currently smoking or were known to have smoked within a year before the interview or death; all the others were classified as non-smokers. Physical activity was scored as minimal for people taking no regular exercise, modest for subjects exercising regulary but no more than 1 day a week and moderate for people taking regular exercise 2-4 times a week. The interviews in all centres were conducted by trained personnel who were familiar with this type of questionnaire.

Parents attended a metabolic ward in the morning and had a general physical examination and a 12-lead ECG at rest which was coded for ischaemic heart disease (as for Minnesota code 1.1, -1.3, 4.1-4.4, 5.1-5.3, 7.1) [16]. Supine blood pressure was measured twice to the nearest $2 \mathrm{mmHg}$ after at least $5 \mathrm{~min}$ rest. Body mass index (BMI) was calculated as weight (in $\mathrm{kg}$ ) divided by height (in $\mathrm{m}^{2}$ ). 
Fasting venous blood was sampled from an antecubital vein for the measurement of serum creatinine with the Jaffè reaction-rate method (Hitachi 737 Autoanalyzer), total serum cholesterol (enzymatic method; Cobas Mira, Roche Diagnostica, Welwin Garden City, Herts, UK), HDL cholesterol, serum triglycerides, (enzymatic method, Cobas Roche). Plasma Lp(a) concentration was measured with an ELISA method (Innotest Lp(a), Byk-Sangtec Diagnostica GmbH and Co. KG Dietzenbach, Germany) in 40 parents of IDDM patients with nephropathy and 43 parents of patients with normoalbuminuria only.

In all the parents except two (because of liver cirrhosis and refusal to participate), one for each group, insulin sensitivity was evaluated by the insulin tolerance test (ITT). The ITT was performed at 08.30 hours the morning of the visit in the post-absorptive state according to the method described by Bonora et al. [17]. At the end of the ITT or in the presence of hypoglycaemic symptoms $20 \mathrm{ml}$ of $20 \%$ glucose solution was given intravenously followed by a $30 \mathrm{~min}$ infusion of a $5 \%$ glucose solution. Of the ITT K index $\left(\mathrm{K}_{\mathrm{itt}}\right)$ of insulin sensitivity was calculated as reported previously [17]. Briefly, the K value represents the ratio between 0.693 and $t_{1 / 2}$, where $t_{1 / 2}$ is the time necessary to reduce basal glycaemia by one half. $T_{1 / 2}$ is calculated from the slope of least square regression analysis of all glycaemic concentrations, taken every $3 \mathrm{~min}$, from the third min until the fifteenth min after intravenous insulin injection. There were 10 parents (5 with nephropathy and 5 without nephropathy) who had NIDDM. Those receiving oral antidiabetic therapy stopped pharmacological treatment for $36 \mathrm{~h}$ before the test and two on insulin stopped long-acting insulin for $36 \mathrm{~h}$ and short-acting insulin for $12 \mathrm{~h}$ before the test. All were controlled on diet. In the subjects receiving antihypertensive drugs treatment was withdrawn for $48 \mathrm{~h}$ before the ITT.

Patients and their parents were informed of the purpose of the study and gave their consent to the study which was performed according the Declaration of Helsinki and with the approval of the local ethics committees.

D efinition of terms. Subjects were considered to have arterial hypertension if systolic blood pressure was greater than $140 \mathrm{mmHg}$ and /or diastolic blood pressure was greater than $90 \mathrm{mmHg}$ or were currently receiving antihypertensive treatment according to National High Blood Pressure Education Program [18].

Subjects were classified as having hyperlipidaemia if total serum cholesterol concentration was equal to or greater than $6.0 \mathrm{mmol} / \mathrm{l}$ and/or serum triglycerides equal to or greater than $2.3 \mathrm{mmol} / \mathrm{l}$ or were currently receiving lipid lowering treatment, according to the Report of National Cholesterol Program Expert Panel [19].

Statistical analysis. Comparisons between groups were made by Student's t-test or Mann-Whitney U test appropriate to the distribution of the variable considered. The chi-square test was used to compare discrete variables. The Mantel-Haenszel summary odds ratio was used to estimate the risk of cardiovascular disease and its risk factors, after adjustment for age, sex, duration of diabetes and smoking status. Miettinen's test-based limits were used to establish the confidence interval. A p value less than 0.05 was considered to indicate statistical significance. Data are given as means \pm SD unless otherwise stated.

\section{Results}

IDDM patients whose parents we studied were similar for age, sex and duration of disease. Their AER differed by selection. Patients with nephropathy had
Table 2. Vital status and age of the parents of IDDM patients with or without nephropathy

\begin{tabular}{lll}
\hline Characteristic & $\begin{array}{l}\text { Parents of patients } \\
\text { with nephropathy }\end{array}$ & $\begin{array}{l}\text { Parents of patients } \\
\text { without nephropathy }\end{array}$ \\
\hline $\begin{array}{l}\text { No. (male/female) } \\
\begin{array}{l}\text { No. died } \\
\text { (male/female) (\%) }\end{array}\end{array}$ & $31 / 31$ & $31 / 31$ \\
$\begin{array}{l}\text { No. alive } \\
\text { (male/female) (\%) }\end{array}$ & $\begin{array}{l}\text { 22/24 (74) } \\
\text { Mean (range) age (years) }\end{array}$ & $9 / 4(21)$ \\
$\quad \begin{array}{l}\text { At death } \\
\text { At time of study }\end{array}$ & $58(43-76)$ & $70(50-90)^{\mathrm{a}}$ \\
\hline
\end{tabular}

${ }^{a} p<0.05$

a higher prevalence of hypertension compared to that of patients without nephropathy (71 vs $10 \%$ $p<0.001$ ) but, because of treatment, their actual blood pressures were similar (Table 1). Clinically diagnosed coronary heart disease was present in two patients in the group with nephropathy and in none in the group without nephropathy.

The demographic data of the parents of the two groups of diabetic patients are shown in Table 2. The number of parents who were dead at the time of the study was similar between the two groups but age at death was significantly younger in the parents of IDDM patients with nephropathy: mean (range) 58 $(43-76)$ vs $70(50-90)$ years; $p<0.05$. Fifty percent $(8$ of 16) of the parents of diabetic patients with nephropathy died of cardiovascular disease, (4 from coronary heart disease and 4 from cerebrovascular disease) compared with $23 \%$ of the parents ( 3 of 13 ) of patients without nephropathy (1 from coronary heart disease and 2 from cerebrovascular disease). Living parents were of similar age, but the proportion with cardiovascular disease was higher in the parents of patients with nephropathy: $17 \%$ (8 of 46 of whom had coronary heart disease and 4 cerebrovascular disease) compared to $4 \%$ ( 2 of 49 , both with coronary heart disease) in the parents of the patients without nephropathy. When mortality and morbidity for cardiovascular disease were combined, the total prevalence of cardiovascular disease in the parents of patients with nephropathy was more than three times that in the parents of patients without nephropathy (odds ratio, 3.96: $95 \%$ CI, 1.3 to $12.2 ; p<0.02$ ).

Actual values of arterial blood pressure, serum triglycerides, total cholesterol, HDL cholesterol and body mass index were similar in the two groups of living parents but the parents of the patients with nephropathy had significantly higher plasma lipoprotein (a) levels and a significantly lower $\mathrm{K}_{\mathrm{itt}}$ index of insulin sensitivity compared to the parents of patients without nephropathy (Table 3 ). The prevalence of arterial hypertension and hyperlipidaemia were significantly higher in the parents of diabetic patients with nephropathy (42 vs $14 \% ; p<0.01 ; 49$ vs $26 \% ; p<0.05$ ). 
Table 3. Clinical and biochemical features of parents of IDDM patients with or without nephropathy

\begin{tabular}{|c|c|c|}
\hline Characteristic & $\begin{array}{l}\text { Parents of } \\
\text { patients with } \\
\text { nephropathy }\end{array}$ & $\begin{array}{l}\text { Parents of pa- } \\
\text { tients without } \\
\text { nephropathy }\end{array}$ \\
\hline Body mass index & $29 \pm 3.8$ & $29 \pm 4.7$ \\
\hline Systolic blood pressure $(\mathrm{mm} \mathrm{Hg})$ & $142 \pm 16$ & $138 \pm 16$ \\
\hline Diastolic blood pressure $(\mathrm{mm} \mathrm{Hg})$ & $84 \pm 8$ & $82 \pm 8$ \\
\hline Triglycerides $(\mathrm{mmol} / \mathrm{l})$ & $1.6 \pm 1.3$ & $1.5 \pm 0.8$ \\
\hline Total cholesterol (mmol/l) & $5.3 \pm 1$ & $5.3 \pm 1.1$ \\
\hline HDL cholesterol (mmol/l) & $1.2 \pm 0.5$ & $1.3 \pm 0.5$ \\
\hline $\mathrm{Lp}(\mathrm{a})(\mathrm{mg} / \mathrm{dl})^{\mathrm{a}}$ & $27.2(1-107)$ & $15.6(0.2-98)^{\mathrm{b}}$ \\
\hline $\mathrm{K}_{\mathrm{itt}} \%$ per $\min ^{\mathrm{a}}$ & $3.7(0.7-6.2)$ & $4.8(0.7-6.7)^{b}$ \\
\hline
\end{tabular}

Table 4. Frequency of arterial hypertension, hyperlipidaemia, diabetes and smoking among parents of IDDM patients with or without nephropathy

\begin{tabular}{lll}
\hline Characteristic & $\begin{array}{l}\text { Parents of } \\
\text { patients with } \\
\text { nephropathy }\end{array}$ & $\begin{array}{l}\text { Parents of } \\
\text { patients without } \\
\text { nephropathy }\end{array}$ \\
\hline Hypertension (\%) & 42 & $14^{\mathrm{b}}$ \\
Hyperlipidaemia (\%) & 49 & $26^{\mathrm{a}}$ \\
Diabetes (\%) & 11 & 10 \\
Smokers (\%) & 29 & 30 \\
\hline
\end{tabular}

${ }^{\mathrm{a}} \mathrm{p}<0.05 ;{ }^{\mathrm{b}} \mathrm{p}<0.01$

Among parents of group A 18 were on antihypertensive treatment and 19 on lipid lowering therapy compared to 6 and 6 subjects respectively among the parents of group B. The frequency of diabetes was similar as was the proportion of smokers between the two parental groups (Table 4). The level of physical activity did not differ between the two parental groups.

In the living parents of diabetic patients with nephropathy the frequency of one or more risk factors for cardiovascular disease (hyperlipidaemia, and/or arterial hypertension, and/or lipoprotein (a) greater than the upper limit of normal of $30 \mathrm{mg} / \mathrm{dl}$; and/or $\mathrm{K}_{\text {itt }}$ lower than the median value for all parents of $4.0 \%$ per min) was more than 5 times that in the parents of the diabetic patients without nephropathy (odds ratio 5.11, $95 \%$ CI 1.4-18.2; $p<0.02$ ).

\section{Discussion}

IDDM patients with albuminuria have an excess incidence of cardiovascular disease morbidity and mortality. This is unlikely to be solely due to the cumulative deleterious effect of risk factors associated with diabetic nephropathy because in this case cardiovascular disease would appear early in most patients with nephropathy. Previous studies have shown that diabetic nephropathy, though likely to affect the progression of pre-existing vascular lesions, probably has no effect on the initiation of the atherosclerotic process [20]. In the present study we confirm, in a different set of Caucasian IDDM patients from the south of Europe, our and other authors' previous observations that a parental history of cardiovascular disease and arterial hypertension [7, 8, 21] is significantly associated with nephropathy in IDDM offspring. Moreover in this study for the first time we were able to identify in the living parents of IDDM patients with nephropathy a significantly higher frequency of risk factors for cardiovascular disease. This was despite death occurring at an earlier age in the parents of IDDM patients with nephropathy, an event which may have removed high risk individuals. Thus the difference in the prevalence of cardiovascular disease risk factors should be taken as a minimum estimate.

Living parents of IDDM patients with nephropathy had more hyperlipidaemia and higher levels of $\mathrm{Lp}(\mathrm{a})$. These abnormalities are recognised independent risk factors for cardiovascular disease in the general population and there is evidence that inheritance plays a key role in the determining levels of a number of lipid fractions $[22,23]$. These results are in general accord with another preliminary study which showed lipid abnormalities in non-diabetic first degree relatives of IDDM patients with microalbuminuria [10]. It is of interest moreover that lipid disturbances in IDDM patients with normoalbuminuria appear to be predictive of the development of microalbuminuria [24]. All these data are consistent with the view that lipid abnormalities in the family and in the patient play a role in the development of diabetic nephropathy and its associated cardiovascular complications.

Reduced insulin sensitivity has been reported both in diabetic patients with established nephropathy [25] and in IDDM patients with microalbuminuria [4]. It was unclear, however, whether the disturbed insulin metabolism was a consequence or a precursor of renal dysfunction. The finding that parents of IDDM patients with nephropathy are more insulin resistant supports the view that changes in insulin sensitivity are likely to precede the development of albuminuria and are part of a metabolic syndrome that may primarily contribute to vascular damage in diabetes. The differences between the two parental groups are unlikely to be the result of an imbalance of environmental factors which can affect insulin sensitivity. In the two groups age distribution was similar and the number of parents with diabetes did not differ significantly. Although regional fat distribution was not measured in this study, body mass index was similar in the two parental groups and as far as it could be assessed there were no differences in physical activity. Our results agree with a recent report which showed reduced insulin sensitivity in those non-diabetic first 
degree relatives of NIDDM patients who had increased AER [26] and with previous family studies which found evidence for insulin resistance, high blood pressure and dyslipidaemia in non-hypertensive, non-diabetic, first degree relatives of patients with NIDDM or essential hypertension [27, 28]. Insulin resistance is considered a risk factor for coronary heart disease in the general population [29] in its own right or through its biochemical sequelae of lipid dysmetabolism and may therefore importantly contribute to explain the excess cardiovascular disease in those diabetic patients who develop albuminuria. Selection bias may potentially affect studies such as ours but several steps were taken to minimize it. All IDDM patients of European origin with persistently elevated AER attending the diabetic clinics over a defined time period and complying with specific criteria for the definition of nephropathy were studied. We believe that these patients are representative of the population of IDDM patients with nephropathy in that most patients who develop albuminuria are expected to attend a specialist clinic. Control patients were selected from the same diabetic population and were matched for age, sex and duration of diabetes. Parents whether dead or alive were assessed in a standardised manner and their clinical or biochemical characteristics were not part of the selection criteria. The rate of ascertainment of information on the parents was $100 \%$ and the two parental groups were similar for age, body mass index and smoking habits. Biochemical determinations and calculation of the $\mathrm{K}_{\mathrm{itt}}$ index were carried out in ignorance of the group to which parents belonged.

It is debatable whether familial aggregation of disease or of risk factors for disease can be explained by familial aggregation of environmental or genetic factors. Analysis in the general population suggests that simple familial clustering of environmental factors is insufficient to account for familial aggregation of disease and that genetic factors are more likely to be implicated [30]. Our findings demonstrating a familial aggregation of risk factors for cardiovascular disease, which operate in the general population, in the subset of IDDM patients who develop nephropathy suggest an inherited susceptibility to atherosclerotic disease in this subgroup. The association between cardiovascular disease risk factors in the family and nephropathy in the diabetic patients supports the view that the process of atherosclerosis and glomerulosclerosis may share common determinants [8] and suggests that therapeutic strategies aimed at correcting the environmental causes contributing to these risk factors may beneficially affect both renal and cardiovascular clinical outcomes.

A cknowledgements. This work was supported by a grant from the Italian Department of Health. Ms. A. Bocci provided invaluable nursing assistance. Part of this work was presented, in abstract form, at the 29th Annual Meeting of the European Association for the Study of Diabetes, Dusseldorf, Germany 1994

\section{References}

1. Borch-Johnsen K, Kreiner S (1987) Proteinuria: value as predictor of cardiovascular mortality in insulin-dependent diabetes mellitus. BMJ 294: 1651-1654

2. Messent JW, Elliott TG, Hill RD, Jarrett RJ, Keen H, Viberti GC (1992) Prognostic significance of microalbuminuria in insulin-dependent diabetes mellitus: a 23 year follow-up study. Kidney Int 41: 836-839

3. Mogensen CE (1984) Microalbuminuria predicts clinical proteinuria and early mortality in maturity onset diabetes. N Engl J Med 310: 356-360

4. Yip J Mattock MB, Morocutti A, Sethi M, Trevisan R, Viberti GC (1993) Insulin-resistance in insulin-dependent diabetic patients with microalbuminuria. Lancet 342: 883 887

5. Vannini P, Ciavarella A, Flamini M et al. (1984) Lipid abnormalities in insulin-dependent diabetic patients with microalbuminuria Diabetes Care 7: 151-154

6. Seaquist ER, Goetz FC, Rich S, Barbosa J (1989) Familial clustering of diabetic kidney disease: evidence for genetic susceptibility to diabetic nephropathy. N Engl J 320: 11611163

7. Viberti GC Keen H, Wiseman MJ (1987) Raised blood pressure in parents of proteinuric insulin-dependent diabetics. BMJ 295: 515-517

8. Earle K, Walker J, Hill C, Viberti GC (1992) Familial clustering of cardiovascular disease in patients with insulin-dependent diabetes and nephropathy. N Engl J Med 326: 673-677

9. Mangili R, Bending JJ, Scott G, Li LK, Gupta A, Viberti GC (1988) Increased sodium-lithium countetransport activity in red blood cells of patients with insulin-dependent diabetes and nephropathy. N Engl J Med 318: 146-150

10.Yip J, Mattock M, Sethi M, Morocutti A, Viberti GC (1993) Insulin resistance in family members of insulin-dependent diabetic patients with microalbuminuria Lancet 341: 369370

11. Chavers BM, Bilous RW, Ellis EN, Steffes MW, Mauer SM (1989) Glomerular lesions and urinary albumin excretion in type 1 diabetes without overt proteinuria. N Engl J Med 320: 966-970

12. Walker JD, Close CF, Jones SL et al. (1992) Glomerular structure in type 1 (insulin-dependent) diabetic patients with normo-and microalbuminuria. Kidney Int 41: 741-748

13. ISTAT (1984) Classificazione delle malattie, traumatismi e cause di morte. IX Revisione, 1975

14. Rose GA (1962) The diagnosis of ischaemic heart pain and intermittent claudication in field surveys. Bull World Organ 27: 645-658

15. Rose GA, Blackburn H, Gillum RF, Prineas RJ (1982) Cardiovascular survey methods. 2nd ed. WHO Monograph Series, No 56 World Health Organization, Geneva

16. Reid DD, Brett GZ, Hamilton PJS, Jarrett RJ, Keen H, Rose G (1974) Cardiorespiratory disease and diabetes among middel-aged civil servants. A study of screening and intervention. Lancet 1: 469-471

17. Bonora E, Moghetti P, Zancanaro C et al. (1989) Estimates of in vivo insulin action in man: comparison of insulin tolerance test with euglycaemic and hyperglycaemic glucose clamp studies. J Clin Endocrinol Met 68: 374-378 
18. National High Blood Pressure Education Program (1993) Fifth Report of the Joint National Committee on Detection, Evaluation, and Treatment of High Blood Pressure, Bethesda, Md: National Heart, Lung and Blood Institute. U.S. Department of Health Education and Welfare publication NIH 93-1088

19. National Cholesterol Program (1988) Report of the National Cholesterol Program Expert Panel on Detection, Evaluation and Treatment of High Blood Cholesterol in Adults. Arch Intern Med 148: 36

20. Krolewski AS, Kosinski EJ, Waram JH et al. (1987) Magnitude and determinants of coronary artery disease in juvenile-onset insulin-dependent diabetes mellitus. Am J Cardiol 59: 750-755

21. Krolewski AS, Canessa M, Warram JH et al. (1988) Predisposition to hypertension and susceptibility to renal disease in insulin-dependent diabetes mellitus. N Engl J Med 318: 140-145

22. Schaefer EJ, Lamon-Fava S, Jenner JL et al. (1984) Lipoprotein (a) levels and risk of coronary heart disease in men. JAMA 271: 99-1003

23. Durrington PN, Ishola M, Hunt L, Arrol S, Bhatnagar D (1988) Apolipoproteins (a), A-1 and B and parental history in men with early onset ischaemic heart disease. Lancet 1 : 1070-1073
24. Coonrod BA, Ellis D, Becker DJ et al. (1993) Predictors of microalbuminuria in individuals with IDDM. Diabetes Care 16: 1376-1383

25. Niskanen L, Laasko M (1993) Insulin resistance is related to albuminuria in patients with type 2 (non-insulin dependent) diabetes mellitus. Metabolism 42: 1541-1545

26. Forsblom CM, Eriksson JG, Ekstrand AV, Teppo AM, Taskinsen R, Groop LC (1995) Insulin resistance and abnormal albumin excretion in non-diabetic first-degree relatives of patients with NIDDM. Diabetologia 38: 363-369

27. Eriksson J, Franssila-Kallunki A, Ekstrand A et al. (1989) Early metabolic defects in persons at increased risk for non-insulin dependent diabetes mellitus. N Eng J Med 321: 337-343

28. Ferrari P, Weidmann P, Show S et al. (1991) Altered insulin sensitivity, hyperinsulinemia, and dyslipidaemia in individuals with a hypertensive parent. Am J Med 91: 589-596

29. Reaven GM (1988) Role of insulin resistance in human disease. Diabetes 37: 1595-1607

30. Khoury MJ, Beaty TH, Liang K-Y (1988) Can familial aggregation of disease be explained by familial aggregation of environmental risk factors? Am J Epidemiol 127: 674683 